\title{
Understanding the Abrupt Climate Change in the Mid-1970s from a Phase-Space Transform Perspective
}

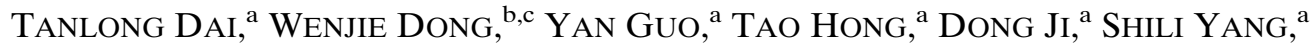 \\ Di TIAN, ${ }^{\mathrm{d}}$ XiAOHANG WEN, ${ }^{\mathrm{e}}$ AND XiAn ZHU ${ }^{\mathrm{a}, \mathrm{c}}$ \\ ${ }^{a}$ State Key Laboratory of Earth Surface Processes and Resource Ecology, Beijing Normal University, Beijing, China \\ ${ }^{\mathrm{b}}$ School of Atmospheric Sciences, Sun Yat-Sen University, Guangzhou, China \\ ${ }^{\mathrm{c}}$ Zhuhai Joint Innovative Center for Climate-Environment-Ecosystem, Future Earth Research Institute, \\ Beijing Normal University, Zhuhai, China \\ ${ }^{\mathrm{d}}$ State Key Laboratory of Satellite Ocean Environment Dynamics, Second Institute of Oceanography, Hangzhou, China \\ ${ }^{\mathrm{e}}$ Plateau Atmosphere and Environment Key Laboratory of Sichuan Province, College of Atmospheric Sciences, \\ Chengdu University of Information Technology, Chengdu, China
}

(Manuscript received 12 December 2017, in final form 1 June 2018)

\begin{abstract}
Abrupt climate change may cause heat, drought, and flood disasters. In this study, we find that many climate factors [e.g., the East Asian summer monsoon (EASM), the Arctic Oscillation (AO) and the Pacific decadal oscillation (PDO)] show a decadal-scale abrupt change in the 1970s. To analyze this phenomenon thoroughly, a new method of pedigree clustering combined with phase-space analysis (PCPSA) is used to establish two-dimensional phase-space coordinate systems of EASM-AO, EASM-PDO, and AO-PDO and the three-dimensional phase-space coordinate system of EASM-AO-PDO. By using the PCPSA method, it is found that all of the phase-space coordinate systems have a significant abrupt change in the mid-1970s, with a transition period, and the fit to the abrupt change of the phase-space coordinate system is better than $80 \%$, which indicates excellent fit. By analyzing the correlation of EASM, AO, and PDO with sea level pressure (SLP) and sea surface temperature (SST), it is found that SLP has an obvious weakening trend in the high latitudes and an increasing trend in the tropics while SST has an increasing trend in most of the Southern Hemisphere waters and a minor weakening trend in the North Pacific Ocean waters between $30^{\circ}$ and $40^{\circ} \mathrm{N}$. Therefore, the abrupt climate change of the 1970 s may well be a global abrupt change of the climatic system.
\end{abstract}

\section{Introduction}

Extreme weather events have become more frequent since the twentieth century (Yi et al. 2015). Abrupt climate change often leads to heat, drought, and flood disasters (Huang et al. 2017a,b). In recent years, against the background of global warming, the surface air temperature has shown a warming hiatus (Huang et al. 2017c). Climate change, especially abrupt climate change, has been the subject of much research since the 1990s. Thus, there is a significant need for the analysis of abrupt climate change.

The definition of climate change given by Fu and Wang in a series of studies on abrupt climate change in the 1990s refers to the climate jumping from one stable state to another (Fu and Wang 1992). Wang (2001) pointed out that a much larger abrupt climate change happened

Corresponding author: Wenjie Dong, dongwj3@mail.sysu.edu.cn around the 1970s, with temperature, air pressure, wind field, and rainfall climate factors all showing the same change (Wang 2001). Many researchers have demonstrated that the 1970s climate change over East Asia is an interdecadal change associated with the coupled oceanatmosphere system (Zhou et al. 2009). Kachi and Nitta (1997) investigated sea surface temperature (SST) variability on a global scale and found interdecadal variability, particularly in the North Pacific; their study also shows an abrupt change of the atmosphere-ocean system in the Pacific Ocean in the mid-1970s, corresponding to that in global surface temperature. The concept of the Pacific decadal oscillation (PDO) has been proposed, with abrupt interdecadal change identified in the years 1890, 1925, 1947, and 1976 (Biondi et al. 2001). The SST of the Pacific Ocean is characterized by the PDO mode at various times, and it is one of the important components of interdecadal change in the SST field (Xiao and Li 2007). Recent studies have shown that the East Asian 
winter monsoon (EAWM) had a weakening trend at the end of the 1970s (Hsu 2016; Tao and Chen 1987). The East Asian summer monsoon (EASM) and EAWM have a direct effect on the climate of China and the whole East Asian region (Zhu et al. 2016). Cheung et al. (2016) pointed out that the Arctic Oscillation (AO) underwent a phase transformation in 1978 (Cheung et al. 2016). The AO is an important atmospheric circulation teleconnection pattern in the high latitudes of the Northern Hemisphere, influenced by mass changes in the mid- to high-latitude atmosphere and forming a seesaw pattern, which in turn controls and affects many extreme weather events in the Northern Hemisphere (Thompson and Wallace 1998). Later studies have shown a relationship between the PDO, EASM, and AO. There is a consistent abrupt change in the EASM and PDO in the 1970s, which may have affected the change in summer rainfall in East China after the 1970s (Chen et al. 2015b; Zhu et al. 2015). In addition, the forcing to PDO to the decadal change of East Asian summer monsoon since the 1970s have demonstrated by both long-term data diagnosis and numerical modeling ( $\mathrm{Li}$ et al. 2010; Qian and Zhou 2014). A correlation between the AO and PDO has been reported previously (Sun and Wang 2006). Therefore, it is important to study abrupt climate change with EASM, AO, and PDO representing the climate. Because of air-sea interaction, air temperatures in North America, near the equator, the Arctic and the HuangHuai-Hai region in China were also affected by obvious abrupt change in 1976 (Ma and Fu 2007; Yin et al. 2015). Jin et al. (2016) reported an abrupt interdecadal change in the mei-yu rainfall, global precipitation, and local precipitation in 1976, which shows correlation with the PDO (Jin et al. 2016; Wang et al. 2016; Wu 2016). Data analyses have revealed a long-term change of global land monsoon precipitation. Numerical model experiments have demonstrated that this kind of change is driven by PDOlike tropical ocean warming (Zhou et al. 2008a,b). In addition, a consistent abrupt change of El Niño-Southern Oscillation (ENSO) and the PDO in the 1970s has been found (Cai et al. 2017). Under the influence of recent human activity, radiative forcing has also exhibited consistent abrupt change with the PDO (Zhu et al. 2016). Wang and $\mathrm{He}$ (2012) noted that there is some correlation between the interdecadal change of the EAWM and ENSO in the 1970s (Wang and He 2012). Besides, the coupling of ENSO with Asian-Australian summer monsoon has been enhanced since 1970s (Wang et al. 2008). Other atmospheric features display the same abrupt interdecadal change, for example, the Aleutian low (AL), the western Pacific subtropical high (WPSH), and the North Atlantic Oscillation (NAO) (Chen et al. 2015a; Weisheimer et al. 2017; Zuo et al. 2016). The above
TABLE 1. The major abrupt climate changes.

\begin{tabular}{cl}
\hline $\begin{array}{c}\text { Acronym of the } \\
\text { abrupt climate change }\end{array}$ & \multicolumn{1}{c}{$\begin{array}{c}\text { Full name of the } \\
\text { abrupt climate change }\end{array}$} \\
\hline PDO & $\begin{array}{l}\text { The Pacific decadal oscillation } \\
\text { The Arctic Oscillation } \\
\text { AO }\end{array}$ \\
The East Asian summer monsoon \\
EAWM & The East Asian winter monsoon \\
ENSO & El Niño-Southern Oscillation \\
NAO & The North Atlantic Oscillation \\
AL & The Aleutian low \\
WPSH & The western Pacific subtropical high \\
\hline
\end{tabular}

studies all suggest that an abrupt change in the climate system occurred in the 1970s, and in this context Yan et al. have studied abrupt climate system change using a nonlinear mathematical approach (Yan et al. 2015, 2016).

All in all, there are many abrupt climate changes as mentioned above, and the major abrupt climate changes are presented in Table 1. However, because of a lack of systematic research, the differences in timing of the abrupt changes, and the fact that most studies of abrupt climate change were based on one-dimensional time series of different variables, abrupt change of the complex climate system has not been explained satisfactorily. In this study we explore the abrupt climate change of the 1970s, taking account of previous research but using a new method of pedigree clustering that combines phasespace analysis. The remainder of this paper is organized as follows. The datasets, methods, and indices are described in section 2. Section 3 shows the results, including determining and testing the time of abrupt change of EASM, AO, and PDO; describing and analyzing the abrupt change of the phase space composed of EASM, AO, and PDO; and analyzing the correlation of EASM, AO, and PDO with sea level pressure (SLP) and SST. Section 4 discusses the results and presents the conclusions.

\section{Datasets and methods}

This study uses monthly mean SST and SLP datasets from the National Oceanic and Atmospheric Administration Extended Reconstructed SST, version 3b (ERSSTv3b), data (Smith et al. 2008) and the National Centers for Environmental Prediction-National Center for Atmospheric Research (NCEP-NCAR) reanalysis SLP (Kalnay et al. 1996). The ERSSTv3b dataset has $2^{\circ} \times 2^{\circ}$ horizontal resolution and the SLP has a $2.5^{\circ} \times$ $2.5^{\circ}$ horizontal resolution. To compare with subsequent model simulation results, we selected the time period 1948-2005. To ensure the consistency of the data resolution, the NOAA Extended Reconstructed SST is interpolated to a standard $2.5^{\circ} \times 2.5^{\circ}$ grid in this analysis. 
The EASM index (EASMI) is defined as follows. We calculate the seasonal average SLP at seven latitudes from $20^{\circ}$ to $50^{\circ} \mathrm{N}$ at $110^{\circ}$ and $160^{\circ} \mathrm{E}$, and similarly for the SLP difference and sum below. Then we calculate the standardized difference of SLP between $110^{\circ}$ and $160^{\circ} \mathrm{E}$ over $20^{\circ}-50^{\circ} \mathrm{N}$ (seven latitudes). Then we the sum the standardized sea level pressure of $20^{\circ}-50^{\circ} \mathrm{N}$ (seven latitudes). Last, we standardize the result again (Shi and Zhu 1996). The AO index is defined as the principal component corresponding to the leading empirical orthogonal function mode of SLP anomalies over the Northern Hemisphere from $20^{\circ}$ to $90^{\circ} \mathrm{N}$ (Thompson and Wallace 1998). The PDO index is derived as the leading principal component of the monthly SST anomalies in the North Pacific Ocean north of $20^{\circ} \mathrm{N}$ (Mantua et al. 1997). In this study, we validate and determine the abrupt change of the EASM, AO, and PDO in the 1970s using the moving $t$-test technique (MTT). The MTT is used to detect abrupt change through examining whether the difference between the mean values of two subsamples is significant. We can obtain the $t_{0}$ statistic sequence by using MTT to determine the sequence of a certain moment. In Fig. 1, the abrupt change years are the year corresponding to the maximum or minimum when $\left|t_{0}\right|<t_{\alpha}$; these are also referred to as abrupt changepoints or transition years (Jiang et al. 2002; Xiao and $\mathrm{Li}$ 2007).

In mathematics and physics, phase space is composed of state variables or components of the state vector in a dynamic system and can represent all possible states of the system. Each possible state of the system is represented by a corresponding point in phase space, and we take $p, q$ amount of the state as the coordinates of the system. Moreover, we take the function $T(p, q)$ to characterize travel through phase space with time. We use EASMI, AO, and PDO as coordinates, and $T$ (EASMI, AOI), $T$ (EASM, PDOI), $T$ (AOI, PDOI), and $T$ (EASMI, AOI, PDOI) as the functions of phase space, which move with time. Following previous studies, we take 1976 as the boundary year, with $1948-75$ as the period before abrupt change and 1976-2005 as the period after abrupt change.

We use the optimal focus distance classification from the spectrum clustering method. Classification results are presented in Table 2, and we define the following five classification parameters:

1) center before abrupt change (CBAC) is the center of gravity of the classification results before the abrupt change,

2) center after abrupt change (CAAC) is the center of gravity of the classification results after the abrupt change,

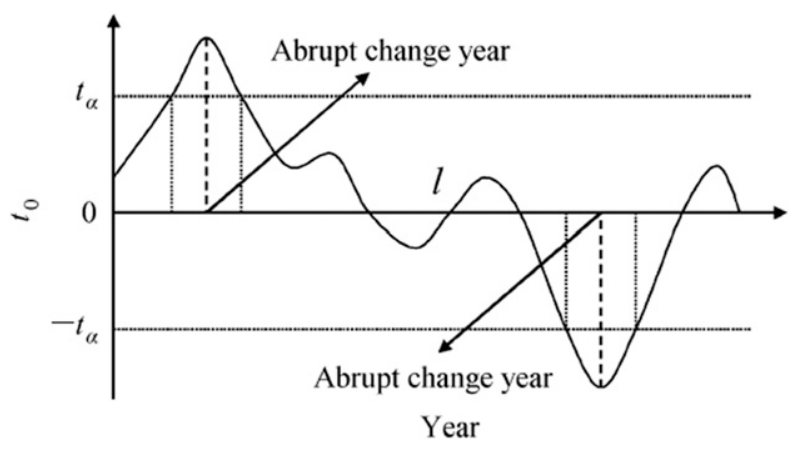

FIG. 1. Schematic diagram of the moving $t$ statistic interdecadal abrupt change. Curve $l$ is the sequence of the statistic $t_{0}$. The threshold $\alpha$ sets the confidence level for abrupt change.

3) area of the abrupt change (AAC) is the area formed by the circle or sphere with radius equal to the distance from the CBAC or CAAC to the origin, and 4) spill point (SP) is the point outside the AAC.

5) the fit of the phase space to the abrupt change, or $\varepsilon$, is $\varepsilon=\left(n_{1} / N_{1}\right) \times 50 \%+\left(n_{2} / N_{2}\right) \times 50 \%$, where $n_{1}$ is the number of years classified as before the abrupt change, $N_{1}$ is the number of years in the period 1948-75 before abrupt change, $n_{2}$ is the number of years classified as after abrupt change, and $N_{2}$ is the number of years in the period 1976-2005 after the abrupt change.

The last classification parameter, $\varepsilon$, characterizes the ability of phase space to represent the abrupt change, that is to say, the fit to the climate factors combined into the phase space with the abrupt change in the phase space. In this study, $\varepsilon$ is divided into four classes:

1) when $\varepsilon<70 \%$, the fit to the abrupt change of the phase space is poor-that is to say, the abrupt change does not occur in the phase space,

2) when $70 \% \leq \varepsilon<80 \%$, the fit to the abrupt change of the phase space is good-that is to say, the abrupt change can happen in the phase space,

3) when $80 \% \leq \varepsilon<90 \%$, the fit to the abrupt change of the phase space is excellent - that is to say, the abrupt change is very likely to happen in the phase space, and

4) when $90 \% \leq \varepsilon<100 \%$, the fit to the abrupt change of the phase space is perfect-that is to say, the abrupt change almost always happens in the phase space.

We calculate the $\varepsilon$ of every phase space shown in Table 3. Spectral clustering is a clustering method that is based on graph theory, in which the weighted undirected graph is divided into $\geq 2$ suboptimal figures, such that elements inside the subfigures are as close as possible but the distance between the subfigures is as great as 


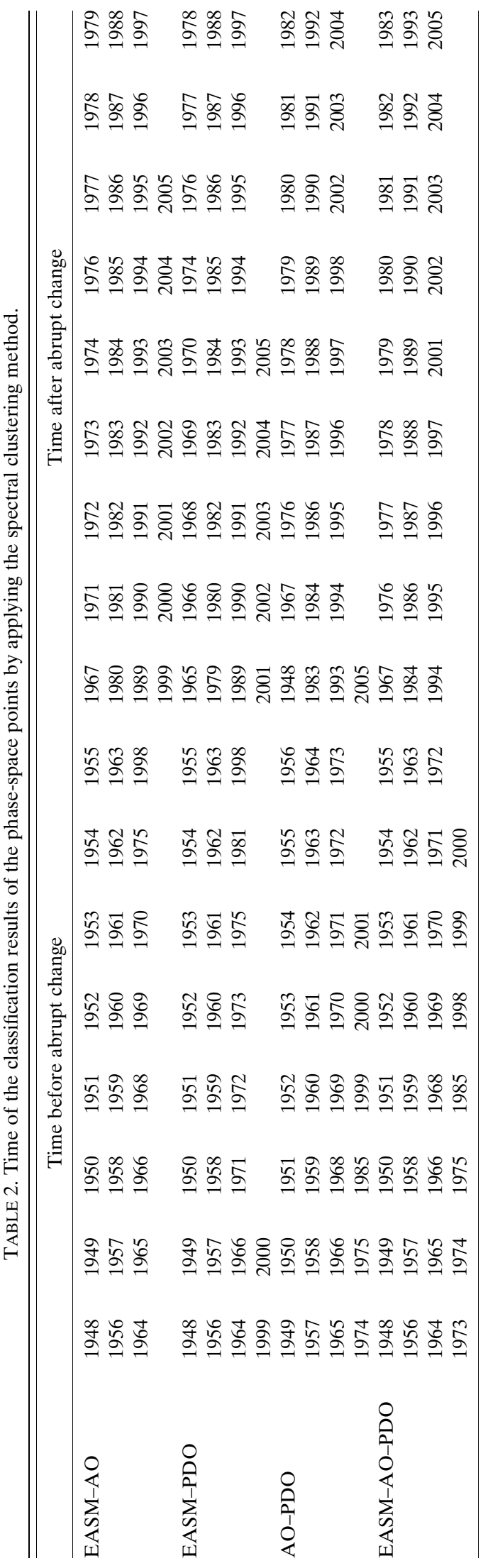

possible so as to achieve common clustering (Chen and Cai 2011). A class sample of the spectrum level is specified as $G$, and the sum of squares within the class $G_{k}$ is

$$
\begin{aligned}
S_{k} & =\sum_{i \in G_{k}}\left(x_{i}-\bar{x}_{k}\right)^{\mathrm{T}}\left(x_{i}-\bar{x}_{k}\right) \\
& =\sum_{i \in G_{k}}\left|x_{i}-\bar{x}_{k}\right|^{2},
\end{aligned}
$$

where $\bar{x}_{k}$ is the center of gravity of class $G_{k}$, and the smaller $S_{k}$ is, the more similar is each sample of $G_{k}$. There are $G$ classes in the $G$ level of the spectrum, which is defined as

$$
P_{G}=\sum_{k=1}^{G} S_{k}
$$

In addition, we take $T$ as the sum of squared residuals of all samples:

$$
\begin{aligned}
T & =\sum_{i=1}^{n}\left(x_{i}-\bar{x}\right)^{\mathrm{T}}\left(x_{i}-\bar{x}\right) \\
& =\sum_{i=1}^{n}\left|x_{i}-\bar{x}\right|^{2}
\end{aligned}
$$

and define the statistic $R^{2}$ as

$$
R^{2}=1-\left(P_{G} / T\right)
$$

$R^{2}$ can be used to evaluate the efficacy of the clustering of each merger; it is obvious that $0 \leq R \leq 1$. We recognize the following three conditions:

1) when each sample of $n$ samples becomes each class, $R^{2}=1$,

2) When $n$ samples merge into a class, $R^{2}=0$, and

3) the value of $R^{2}$ always decreases with a reduction in the number of categories, and we take the class before the class that rapidly declines.

\section{Results}

We calculated the EASM index, AO index, and PDO index, and the corresponding time series are shown in Figs. 2a, 2c, and 2e, respectively. Figure 2a shows an obvious abrupt change of EASMI during the 1970s, from positive before 1965 to almost always negative after 1975, with a transition period in 1965-75 when the EASMI takes negative or positive values. Thus, the EASM has a relatively long transition period for the abrupt change in the 1970s. Figure 2c shows an obvious abrupt change of the AO during the 1970s, from 
TABLE 3. Goodness of fit to abrupt change of the phase space $\varepsilon$ for the four phase spaces considered.

\begin{tabular}{lc}
\hline \hline Type of phase space & $\begin{array}{c}\text { Fit to abrupt change of } \\
\text { the phase space } \varepsilon\end{array}$ \\
\hline EASM-AO & $89.4 \%$ \\
EASM-PDO & $82.6 \%$ \\
AO-PDO & $89.8 \%$ \\
EASM-AO-PDO & $91.5 \%$ \\
\hline
\end{tabular}

negative before 1972 to almost always positive after 1975. The AO shows a very short transition period for the abrupt change in the 1970s. Figure 2e shows an obvious abrupt change of the PDO during the 1970s, from negative before 1975 to almost always positive after 1975. Note that the PDO is positive in 1957-61 and negative in 1997-2002. Thus, PDO shows a short transition period, but has a greater interdecadal disturbance in the 1970s. In this paper, we assess the timing of the abrupt change and its significance by applying the MTT method. As can be seen from the figures, most of the abrupt changepoints of EASM and AO, as tested by MTT, do not occur in the 1970s. However, the actual time of the abrupt change is significant at the $95 \%$ level, and therefore using the time series we identify clear abrupt changes of EASM, AO, and PDO in the 1970s.

To better study abrupt climate change, in this paper we use a phase-space approach combined with hierarchical clustering analysis to establish the two- and three-dimensional phase spaces shown in Figs. 3 and 4. From Figs. $3 a-c$ and 4 we can see that there is an
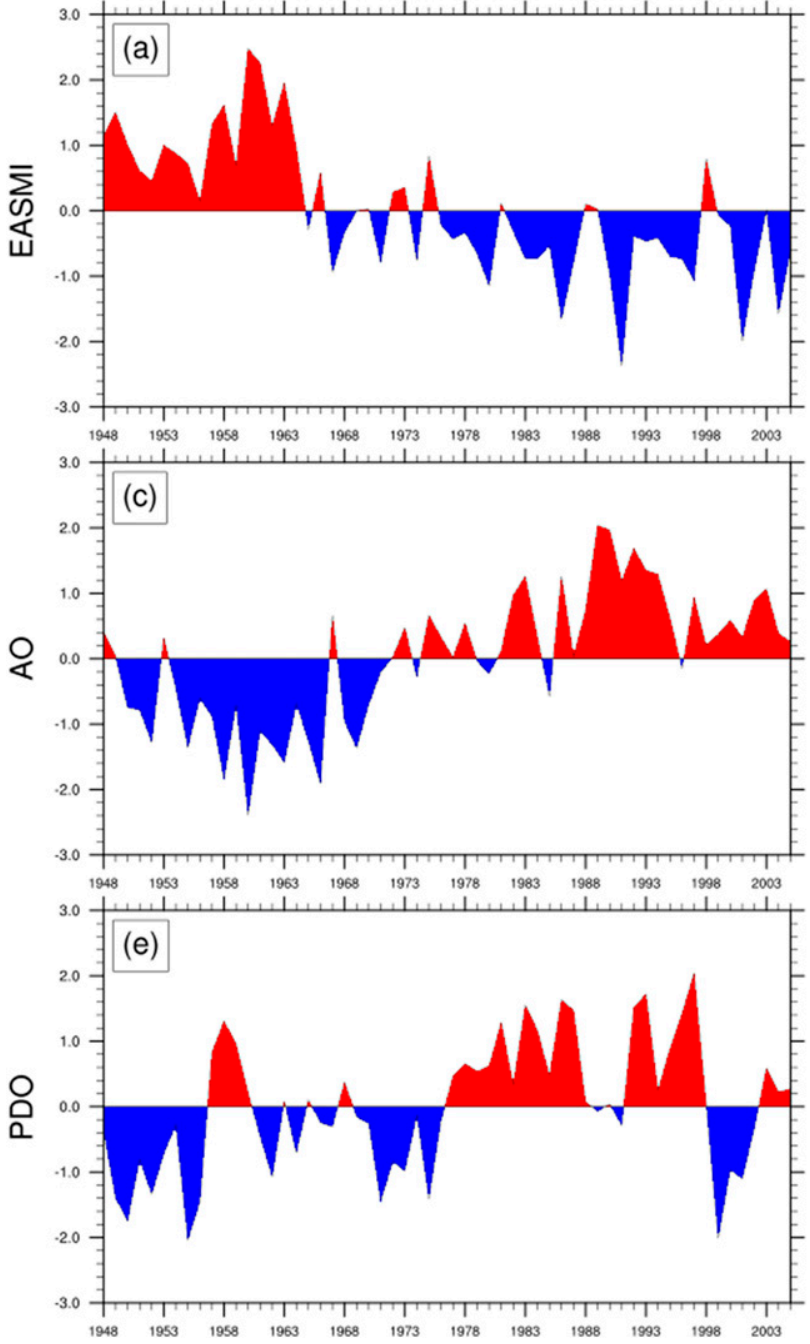
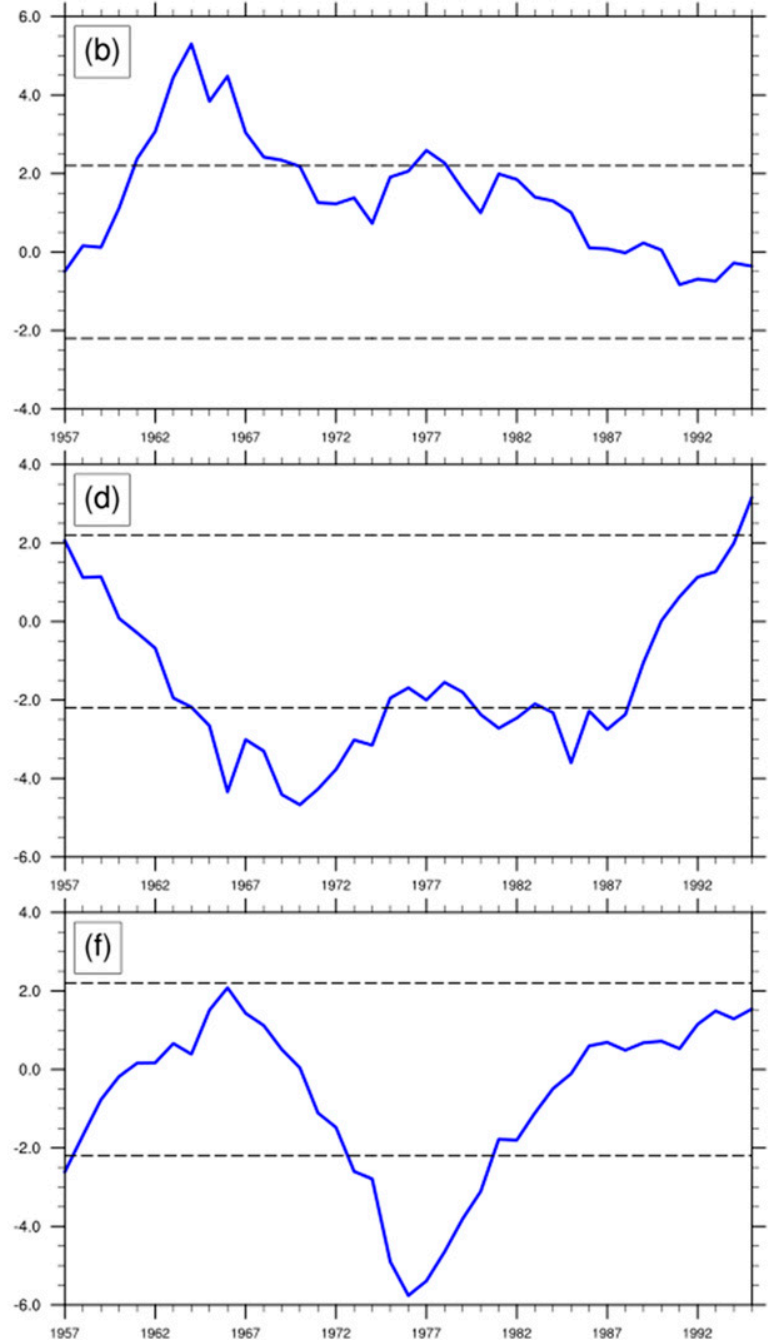

FIG. 2. The 1948-2005 time series of (a) EASMI, (c) AO, and (e) PDO, along with the results of applying the MTT to the (b) EASMI, (d) AO, and (f) PDO time series. The horizontal dashed lines denote the $95 \%$ confidence level according to MTT. 

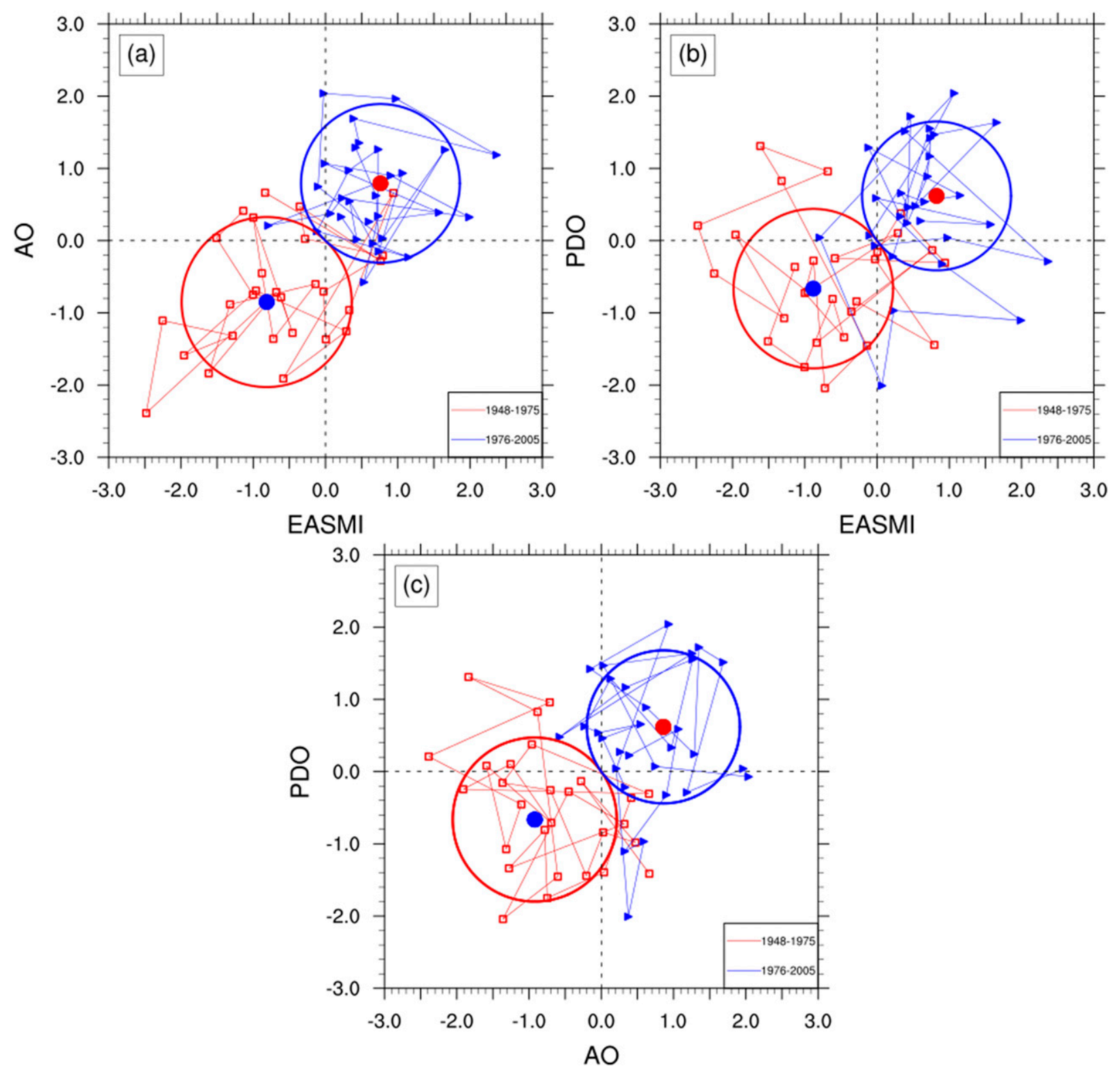

FIG. 3. Two-dimensional phase-space diagrams for the phase spaces composed of (a) EASM and AO, (b) EASM and PDO, and (c) AO and PDO. The red and blue dots represent the center before and after, respectively, the abrupt change. The open circles indicate areas of abrupt change. Red lines and symbols represent the points for 1948-75; blue lines and symbols represent the points for 1976-2005.

obvious abrupt change in every phase space, from the 1948-75 period to the 1976-2005 period. In addition, the points within every phase space are all concentrated near the $\mathrm{CBAC}$ and CAAC, and $\varepsilon$ is more than $80.0 \%$; that is, an excellent fit of the abrupt change. In addition, in Figs. 3a, 3c, and 4, the number of spill points is very small, meaning little disturbance in that period. Moreover, the transition period is short. In Fig. 3b, however, the number of spill points is slightly greater than in the previous cases, which means some minor disturbance in that period. Moreover, the transition period is a little longer and $\varepsilon$ is the least.

In this paper, we judge and analyze the obvious abrupt change of the climate system in the 1970s, taking EASM, $\mathrm{AO}$, and PDO as examples. To explore the reasons for the abrupt climate change, we analyze the correlations of EASM, AO, and PDO with SLP (Figs. 5a,c,e) and SST (Figs. 5b,d,f). There is a significant negative correlation of EASM with SLP (Fig. 5a) in mid-low latitudes. However, the correlation is significantly positive in the 


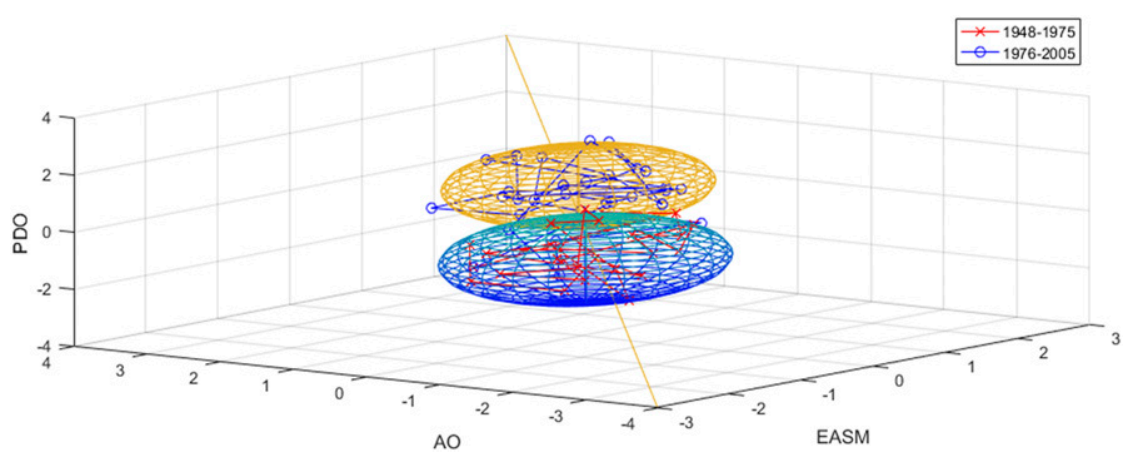

FIG. 4. Three-dimensional phase-space diagram composed of EASM, AO, and PDO. The red lines and symbols represent the points in the 1948-75 period, and the blue lines and symbols represent the points in the 1976-2005 period. Blue and yellow spheres represent the areas before and after, respectively, the abrupt change. The yellow line represents a diagonal of the cube.

polar latitudes. There is a strongly negative significant correlation between EASM and SST (Fig. 5b) in tropical waters, most of the Southern Hemisphere. Figure 5c shows a significant negative correlation of AO with SLP in polar latitudes, while their correlation is very significantly positive in mid-low latitudes. There is a positive significant correlation of AO with SST (Fig. 5d) in most region. Figure 5e shows a significant negative correlation between PDO and SLP in most parts of the eastern Pacific Ocean and most areas south of $60^{\circ} \mathrm{N}$. Conversely, their correlation is clearly positive in most tropical regions and near Australia. Figure $5 \mathrm{f}$ shows a positive significant correlation of PDO and SST between $30^{\circ}$ and $50^{\circ} \mathrm{N}$ in the Pacific Ocean, and their correlation is obviously positive in tropical waters, most waters of the Southern Hemisphere and rare sea areas of the Northern Hemisphere. The abrupt climate change of the 1970s is likely to be a climate system mutation on a global scale, influenced by air-sea interaction and a complex interplay of the circulations of the atmosphere and ocean.

\section{Conclusions and discussion}

We calculated the EASM index, AO index, and PDO index using NOAA extended reconstructed SST and NCEP-NCAR reanalysis data. We used the MTT to detect the years of abrupt decadal change and obtained results consistent with a number of studies; that is, EASM, AO, and PDO show significant abrupt changes in the 1970s.

We established two-dimensional phase spaces and a three-dimensional phase space based on the three indices (EASM-AO, EASM-PDO, AO-PDO, and EASMAO-PDO), and combined the phase-space approach with spectral clustering. The results, together with the analysis of $\varepsilon$, led to the following conclusions.
There is an obvious abrupt change in every phase space, from the 1948-75 period to the 1976-2005 period. In addition, the points within every phase space are all concentrated near the CBAC and CAAC, and $\varepsilon$ is more than $80.0 \%$, that is, an excellent fit of the abrupt change. In addition, in the phase space of the EASM-AO, AOPDO, and EASM-AO-PDO, the number of spill points is very small, meaning little disturbance in that period. Moreover, the transition period is short. In the phase space of the EASM-PDO, however, the number of spill points is slightly greater than in the previous cases, which means some minor disturbance in that period. Moreover, the transition period is a little longer and $\varepsilon$ is the least.

In this study, we confirmed the abrupt climate change in the 1970s, consistent with previous studies, and validated the timing of the abrupt change in the climate system by applying a new phase-space method using spectral clustering. To test for an abrupt change in the global climate system in the 1970s, we analyzed the correlations of EASM, AO, and PDO to SLP and SST. The SLP of the high-latitude Southern Hemisphere shows an obvious weakening trend before and after abrupt climate change, and the SLP of the high-latitude Northern Hemisphere shows an obvious weakening trend; however, the SLP of the high-latitude Northern Hemisphere and tropics shows an obvious increasing trend, especially in Africa, as over most of Asia and western Europe. SST in the Southern Hemisphere waters generally has a stronger increasing trend, with the SST in the Arctic Ocean increasing less, while the SST over $30^{\circ}-40^{\circ} \mathrm{N}$ in the North Pacific Ocean has an obvious weakening trend. The abrupt climate change of the 1970s is likely to have been a global-scale climate system phenomenon, with an interplay between air-sea interaction and the complex effects of the atmospheric and ocean circulation. 

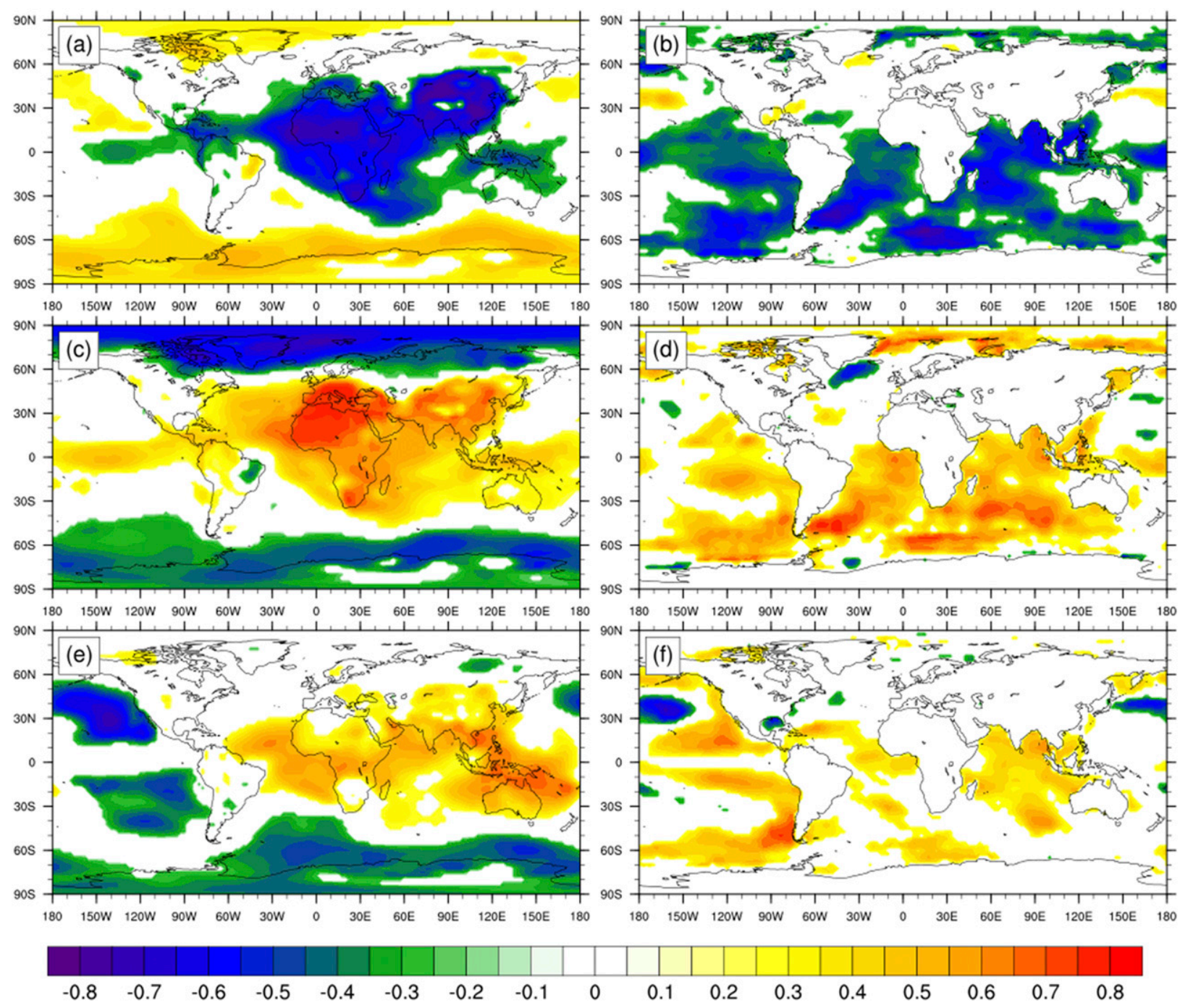

FIG. 5. The correlation between SLP and (a) EASM, (c) AO, and (e) PDO, and the correlation between SST and (b) EASM, (d) AO, and (f) PDO. Color shading indicates significance at the $95 \%$ level or better.

In this paper, we focus on statistical methods to study abrupt climate change and did not analyze the mechanisms from climate dynamics. We used reanalysis data and have not considered model simulation results from CMIP5. Our study has not addressed predictions of future abrupt climate change. These will form the main content of the next stage of our research.

Acknowledgments. This study was funded by the National Key Program of the National Natural Science Foundation of China (41330527) and the National Key Research and Development Program of China (2016YFA0602703). Authors TD, WD, and YG designed the research; TD, TH, and DJ analyzed the data; TD wrote the paper; DT and SY helped to collect the data; and XW and XZ helped to draft the figures. All of the authors participated in the analysis of the results.

\section{REFERENCES}

Biondi, F., A. Gershunov, and D. R. Cayan, 2001: North Pacific decadal climate variability since 1661 . J. Climate, 14, 5-10, https:// doi.org/10.1175/1520-0442(2001)014<0005:NPDCVS>2.0.CO;2.

Cai, J., J. Xu, A. M. Powell, Z. Guan, and L. Li, 2017: Intercomparison of the temperature contrast between the Arctic and equator in the pre- and post periods of the 1976/1977 regime shift. Theor. Appl. Climatol., 127, 761-767, https://doi.org/ 10.1007/s00704-015-1663-z.

Chen, S., B. Yu, and W. Chen, 2015a: An interdecadal change in the influence of the spring Arctic Oscillation on the subsequent ENSO around the early 1970s. Climate Dyn., 44, 1109-1126, https://doi.org/10.1007/s00382-014-2152-2. 
_ W. Chen, and R. Wu, 2015b: An interdecadal change in the relationship between boreal spring Arctic Oscillation and the East Asian summer monsoon around the early 1970s. J. Climate, 28, 1527-1542, https://doi.org/10.1175/JCLI-D-14-00409.1.

Chen, X., and D. Cai, 2011: Large scale spectral clustering with landmark-based representation. Proc. 25th AAAI Conf. on Artificial Intelligence, San Francisco, CA, AAAI, 313-318, https://www.aaai.org/ocs/index.php/AAAI/AAAI11/paper/view/ $3484 / 3883$.

Cheung, H. H. N., W. Zhou, M. Y. T. Leung, C. M. Shun, S. M. Lee, and H. W. Tong, 2016: A strong phase reversal of the Arctic Oscillation in midwinter 2015/2016: Role of the stratospheric polar vortex and tropospheric blocking. J. Geophys. Res. Atmos., 121, 13 443-13 457, https://doi.org/10.1002/2016JD025288.

Fu, C., and Q. Wang, 1992: The definition and detection of the abrupt climatic change. Chin. J. Atmos. Sci, 16, 482-493.

Hsu, P.-C., 2016: Global monsoon in a changing climate. The Monsoons and Climate Change: Observations and Modeling, L. M. Véspoli de Carvalho and C. Jones, Eds., Springer, 7-24.

Huang, J., H. Yu, A. Dai, Y. Wei, and L. Kang, 2017a: Drylands face potential threat under $2^{\circ} \mathrm{C}$ global warming target. Nat. Climate Change, 7, 417-422, https://doi.org/10.1038/nclimate3275.

- , and Coauthors, 2017b: Dryland climate change: Recent progress and challenges. Rev. Geophys., 55, 719-778, https:// doi.org/10.1002/2016RG000550.

—, Y. Xie, X. Guan, D. Li, and F. Ji, 2017c: The dynamics of the warming hiatus over the Northern Hemisphere. Climate Dyn., 48, 429-446, https://doi.org/10.1007/s00382-016-3085-8.

Jiang, S., S. E. Moriarty, H. Grossniklaus, K. C. Nelson, D. P. Jones, and S. P. Jr, 2002: Increased oxidant-induced apoptosis in cultured nondividing human retinal pigment epithelial cells. Invest. Ophthalmol. Visual Sci., 43, 2546-2553.

Jin, H., W. He, Q. Liu, J. Wang, and G. Feng, 2016: The applicability of research on moving cut data-approximate entropy on abrupt climate change detection. Theor. Appl. Climatol., 124, 475-486, https://doi.org/10.1007/s00704-015-1428-8.

Kachi, M., and T. Nitta, 1997: Decadal variations of the global atmosphere-ocean system. J. Meteor. Soc. Japan, 75, 657-675, https://doi.org/10.2151/jmsj1965.75.3_657.

Kalnay, E., and Coauthors, 1996: The NCEP/NCAR 40-Year Reanalysis Project. Bull. Amer. Meteor. Soc., 77, 437-471, https:// doi.org/10.1175/1520-0477(1996)077<0437:TNYRP>2.0.CO;2.

Li, H., A. Dai, T. Zhou, and J. Lu, 2010: Responses of East Asian summer monsoon to historical SST and atmospheric forcing during 1950-2000. Climate Dyn., 34, 501-514, https://doi.org/ 10.1007/s00382-008-0482-7.

Ma, Z., and C. Fu, 2007: Global aridification in the second half of the 20th century and its relationship to large-scale climate background. Sci. China, 50D, 776-788, https://doi.org/10.1007/ s11430-007-0036-6.

Mantua, N. J., S. R. Hare, Y. Zhang, J. M. Wallace, and R. C. Francis, 1997: A Pacific interdecadal climate oscillation with impacts on salmon production. Bull. Amer. Meteor. Soc., 78, 1069-1080, https://doi.org/10.1175/1520-0477(1997)078<1069: APICOW $>2.0 . \mathrm{CO} ; 2$.

Qian, C., and T. Zhou, 2014: Multidecadal variability of north China aridity and its relationship to PDO during 1900-2010. J. Climate, 27, 1210-1222, https://doi.org/10.1175/JCLI-D-13-00235.1.

Shi, N., and Q. Zhu, 1996: An abrupt change in the intensity of the East Asian summer monsoon index and its relationship with temperature and precipitation over East China. Int. J. Climatol., 16, 757-764, https://doi.org/10.1002/(SICI)10970088(199607)16:7<757::AID-JOC50>3.0.CO;2-5.
Smith, T. M., R. W. Reynolds, T. C. Peterson, and J. Lawrimore, 2008: Improvements to NOAA's historical merged landocean surface temperature analysis (1880-2006). J. Climate, 21, 2283-2296, https://doi.org/10.1175/2007JCLI2100.1.

Sun, J., and H. Wang, 2006: Relationship between Arctic Oscillation and Pacific decadal oscillation on decadal timescale. Chin. Sci. Bull., 51, 75-79, https://doi.org/10.1007/s11434-004-0221-3.

Tao, S. Y., and L. X. Chen, 1987: A review of recent research on the East Asian summer monsoon in China. Monsoon Meteorology, C.-P. Chang and T. N. Krishnamurti, Eds., Oxford University Press, 60-92.

Thompson, D. W. J., and J. M. Wallace, 1998: The Arctic oscillation signature in the wintertime geopotential height and temperature fields. Geophys. Res. Lett., 25, 1297-1300, https://doi.org/ 10.1029/98GL00950.

Wang, B., J. Yang, T. Zhou, and B. Wang, 2008: Interdecadal changes in the major modes of Asian-Australian monsoon variability: Strengthening relationship with ENSO since the late 1970s. J. Climate, 21, 1771-1789, https://doi.org/10.1175/ 2007JCLI1981.1.

Wang, H., 2001: The weakening of the Asian monsoon circulation after the end of 1970's. Adv. Atmos. Sci., 18, 376-386, https:// doi.org/10.1007/BF02919316.

_ , and S. He, 2012: Weakening relationship between East Asian winter monsoon and ENSO after mid-1970s. Chin. Sci. Bull., 57, 3535-3540, https://doi.org/10.1007/s11434-012-5285-x.

Wang, S. Y. S., Y.-H. Lin, and C.-H. Wu, 2016: Interdecadal change of the active-phase summer monsoon in East Asia (meiyu) since 1979. Atmos. Sci. Lett., 17, 128-134, https://doi.org/ 10.1002/asl.603.

Weisheimer, A., N. Schaller, C. O'Reilly, D. A. MacLeod, and T. Palmer, 2017: Atmospheric seasonal forecasts of the twentieth century: Multi-decadal variability in predictive skill of the winter North Atlantic Oscillation (NAO) and their potential value for extreme event attribution. Quart. J. Roy. Meteor. Soc., 143, 917-926, https://doi.org/10.1002/qj.2976.

$\mathrm{Wu}, \mathrm{R} ., 2016$ : Possible roles of regional SST anomalies in long-term changes in the relationship between the Indian and Australian summer monsoon rainfall. Theor. Appl. Climatol., 124, 663677, https://doi.org/10.1007/s00704-015-1443-9.

Xiao, D., and J. Li, 2007: Spatial and temporal characteristics of the decadal abrupt changes of global atmosphere-ocean system in the 1970s. J. Geophys. Res., 112, D24S22, https://doi.org/10.1029/ 2007JD008956.

Yan, P. C., G. L. Feng, and W. Hou, 2015: A novel method for analyzing the process of abrupt climate change. Nonlinear Processes Geophys., 22, 249-258, https://doi.org/10.5194/npg22-249-2015.

- W. Hou, and G. Feng, 2016: Transition process of abrupt climate change based on global sea surface temperature over the past century. Nonlinear Processes Geophys., 23, 115-126, https://doi.org/10.5194/npg-23-115-2016.

Yi, C., E. Pendall, and P. Ciais, 2015: Focus on extreme events and the carbon cycle. Environ. Res. Lett., 10, 070201, https://doi.org/ 10.1088/1748-9326/10/7/070201.

Yin, Y., H. Liu, X. Yi, and W. Liu, 2015: Spatiotemporal variation and abrupt change analysis of temperature from 1960 to 2012 in the Huang-Huai-Hai Plain, China. Adv. Meteor., 2015, 643493, http://dx.doi.org/10.1155/2015/643493.

Zhou, T., R. Yu, H. Li, and B. Wang, 2008a: Ocean forcing to changes in global monsoon precipitation over the recent half-century. J. Climate, 21, 3833-3852, https://doi.org/10.1175/ 2008JCLI2067.1. 
_ L. Zhang, and H. Li, 2008b: Changes in global land monsoon area and total rainfall accumulation over the last half century. Geophys. Res. Lett., 35, L16707, https://doi.org/10.1029/ 2008GL034881.

, D. Gong, J. Li, and B. Li, 2009: Detecting and understanding the multi-decadal variability of the East Asian summer monsoon-Recent progress and state of affairs. Meteor. Z., 18, 455-467, https://doi.org/10.1127/0941-2948/2009/0396.

Zhu, Y., H. Wang, J. Ma, T. Wang, and J. Sun, 2015: Contribution of the phase transition of Pacific decadal oscillation to the late 1990s' shift in East China summer rainfall. J. Geophys. Res. Atmos., 120, 8817-8827, https://doi.org/10.1002/2015JD023545.

, T. Wang, and H. Wang, 2016: Relative contribution of the anthropogenic forcing and natural variability to the interdecadal shift of climate during the late 1970s and 1990s. Sci. Bull., 61, 416-424, https://doi.org/10.1007/s11434-016-1012-3.

Zuo, J., H.-L. Ren, W. Li, and L. Wang, 2016: Interdecadal variations in the relationship between the winter North Atlantic oscillation and temperature in south-central China. J. Climate, 29, 7477-7493, https://doi.org/10.1175/JCLI-D-15-0873.1. 